\title{
Philosophiques
}

\section{Démocratie et frontières : le problème de la constitution du demos et le recours à l'histoire}

\section{Dominique Leydet}

Volume 39, numéro 2, automne 2012

Territorialité, identité nationale et justice mondiale

URI : https://id.erudit.org/iderudit/1013694ar

DOI : https://doi.org/10.7202/1013694ar

Aller au sommaire du numéro

\section{Éditeur(s)}

Société de philosophie du Québec

ISSN

0316-2923 (imprimé)

1492-1391 (numérique)

Découvrir la revue

Citer cet article

Leydet, D. (2012). Démocratie et frontières : le problème de la constitution du demos et le recours à l'histoire. Philosophiques, 39(2), 405-419.

https://doi.org/10.7202/1013694ar
Résumé de l'article

La réponse conventionnelle au problème des limites du demos est que la théorie de la démocratie n’a pas les ressources normatives pour le résoudre. Les limites du demos nous sont données par l'histoire, dont nous reconnaissons la contingence, et le travail de légitimation démocratique ne peut s'effectuer qu'a posteriori à partir de ces limites. Les critiques cosmopolitiques de cette position mettent en cause sa prémisse. Selon eux, la théorie de la démocratie nous donne une réponse normative au problème des frontières. Elle consiste non pas dans la recherche vaine d'un principe normatif permettant de les déterminer, mais plutôt dans l'idée d'un demos sans limite, plaidant ainsi en faveur d'institutions juridiques ou démocratiques globales. Mon objectif est de montrer les limites de cette critique cosmopolitique afin d'esquisser un argument en faveur d'une version revue et corrigée de la position conventionnelle qui assume pleinement les conséquences de son recours à l'histoire. 


\title{
Démocratie et frontières: le problème de la constitution du demos et le recours à l'histoire
}

\author{
DOMINIQUE LEYDET \\ leydet.dominique@uqam.ca \\ Université du Québec à Montréal
}

\begin{abstract}
RÉSUMÉ. - La réponse conventionnelle au problème des limites du demos est que la théorie de la démocratie n'a pas les ressources normatives pour le résoudre. Les limites du demos nous sont données par l'histoire, dont nous reconnaissons la contingence, et le travail de légitimation démocratique ne peut s'effectuer qu'a posteriori à partir de ces limites. Les critiques cosmopolitiques de cette position mettent en cause sa prémisse. Selon eux, la théorie de la démocratie nous donne une réponse normative au problème des frontières. Elle consiste non pas dans la recherche vaine d'un principe normatif permettant de les déterminer, mais plutôt dans l'idée d'un demos sans limite, plaidant ainsi en faveur d'institutions juridiques ou démocratiques globales. Mon objectif est de montrer les limites de cette critique cosmopolitique afin d'esquisser un argument en faveur d'une version revue et corrigée de la position conventionnelle qui assume pleinement les conséquences de son recours à l'histoire.
\end{abstract}

\begin{abstract}
The conventional response to the boundary problem is to say that since democratic theory lacks the normative resources to solve it, we must accept boundaries as determined by history. Questions of democratic legitimacy only make sense from within their framework. Cosmopolitan critics contest the premise of this argument. Democratic theory provides a normative answer to the boundary problem : in principle, the demos has no limits. As democrats, we should be working towards the development of global democratic and legal institutions. My objective is to show the limits of this cosmopolitan critique and sketch an argument in favour of a revised version of the conventional position.
\end{abstract}

La démocratie, définie minimalement par les procédures grâce auxquelles un ensemble d'individus sont appelés à s'auto-gouverner, ne semble pas pouvoir offrir de solution au problème logiquement premier de la constitution de cet ensemble. Elle suppose plutôt son existence préalable. Les principes centraux de la théorie démocratique, comme le consentement ou le principe majoritaire, présupposent pour leur application que l'ensemble des individus auxquels on entend les appliquer soit déjà délimité. En d'autres termes, ils ne peuvent eux-mêmes servir à déterminer qui sont ceux qui doivent être inclus dans leur mise en pratique. Ainsi, il n'y aurait pas de principe démocratique qui permette de donner une réponse normative satisfaisante à la question des limites du demos. C'est ce qu'on appelle dans la littérature le problème de la constitution du demos ou, pour reprendre l'expression de Frederick Whelan, le «boundary problem ${ }^{1}$.

1. Whelan, I983. Voir aussi Dahl, I990, p. 45-5I. 
Ce problème a une dimension territoriale dans la mesure où la souveraineté de la communauté politique constituée s'exerce sur un territoire défini. Quand on parle des limites du demos, on fait donc référence à la composition d'une entité, la communauté politique, qui a une extension territoriale déterminée, et c'est dans ce double sens que le terme de frontières ou de limites est entendu.

Si l'on a pu longtemps se satisfaire du «silence» de la théorie normative, c'est parce que l'on tenait pour acquis que l'aventure démocratique avait à se déployer dans les limites de l'État-nation déjà institué. On concevait ainsi cette question comme ayant été « réglée » par l'histoire, c'est-à-dire le plus souvent par le hasard - ou la nécessité — de la force et des armes. Il est plus difficile aujourd'hui de traiter la constitution du demos comme un "déjà donné " dont on ne saurait interroger la légitimité. Le développement de revendications sécessionnistes au cours du dernier tiers $\mathrm{du}_{\mathrm{xx}} \mathrm{e}^{\mathrm{e}}$ siècle a provoqué une première mise en cause du statu quo des frontières. Aujourd'hui, l'intensification des flux migratoires suscite la contestation de la démarcation citoyens/étrangers, membres/non membres.

Ces contestations nous invitent à questionner la façon dont la théorie de la démocratie a voulu s'en remettre à l'histoire et aux faits pour forclore la question de la constitution du demos et pallier ce qui apparaissait comme son incomplétude. Si la philosophie politique, en tant que réflexion normative, se veut d'abord, comme le disait Rousseau, la recherche du droit et de la raison plutôt que celle du fait ${ }^{2}$, il y a dans cette résignation à la factualité de l'histoire comme point de départ de la démocratie quelque chose qui suscite le doute. La philosophie ne renonce-t-elle pas trop tôt dans sa recherche du droit et de la raison?

Pour répondre à cette question, je vais prendre pour point de départ la défense de la position conventionnelle esquissée par Jürgen Habermas dans ses textes des années I990. Il y reprend le constat que la théorie démocratique, plongeant ses racines dans le contractualisme des modernes, ne donne pas de réponse normative à la question de la constitution du demos. Nous devrions ainsi accepter les frontières déjà données comme le résultat de l'histoire, une histoire décrite comme contingente et arbitraire d'un point de vue moral. Le travail de légitimation démocratique ne commencerait qu'à partir de la factualité d'une histoire qui a suivi son cours et doit être justifiée de façon rétroactive.

Cette position est aujourd'hui dénoncée comme cédant trop vite devant les faits. Certains auteurs en acceptent la prémisse (les principes démocratiques ne donnent pas de réponse à la question de la constitution du demos) pour en contester la conséquence (nous devons accepter comme un donné les frontières résultant de l'histoire). Selon cette première critique,

2. Voir le manuscrit de Genève: «Mais je cherche le droit et la raison et ne dispute pas des faits» Rousseau, I964, p. 297. 
l'absence de justification des limites du demos devrait nous conduire non pas à forclore le débat sur ces limites, mais à accepter qu'elles puissent être contestées et à engager avec ceux qui les contestent un dialogue véritable. Autrement dit, loin de mettre hors champ la question de la légitimité du demos, on peut utiliser la lacune normative des théories démocratiques pour mettre en évidence le caractère contestable des limites de tout demos, et plus précisément la démarcation citoyens/étrangers ${ }^{3}$. Le constat de la contingence des frontières produites par l'histoire devient ici la prémisse d'un argument soutenant la contestation de la légitimité démocratique de frontières particulières et les revendications d'inclusion formulées par, et au nom, des noncitoyens. J'entends montrer que la prise en considération de cette première contestation ne doit pas nous mener à rompre radicalement avec la position conventionnelle, mais nous forcer plutôt à assumer pleinement les conséquences de son recours à l'histoire.

De façon plus radicale, d'autres auteurs, que je qualifierai de cosmopolitiques, mettent en cause la prémisse même de la position conventionnelle (la théorie démocratique n'offre pas de réponse au problème des limites du demos). Dans ce cas, il s'agit de montrer que la théorie de la démocratie possède les ressources normatives, non pas pour résoudre le problème des limites, mais bien pour le dissoudre: à la recherche vaine d'un principe normatif permettant de justifier les frontières, on oppose le principe d'un demos sans limite et l'on plaide en faveur d'institutions juridiques ou démocratiques globales.

J'examinerai deux versions de cette position cosmopolitique: la première s'appuie sur le principe de «tous les intérêts affectés "; la seconde mise plutôt sur les implications de l'exigence démocratique de la justification du pouvoir de contrainte. J'essaierai de montrer qu'aucune de ces versions ne montre de façon convaincante que les principes démocratiques de légitimité nous contraignent à adhérer à l'idée d'un demos global. Mon objectif est d'expliquer pourquoi les principes mobilisés par les partisans de la position cosmopolitique demeurent ultimement indéterminés et ne fournissent pas d'issue à ce problème. Cela étant, c'est à une version revue et corrigée de la position conventionnelle que nous devons adhérer.

\section{La position conventionnelle}

C'est surtout dans les années I990 que Habermas s'intéresse au rapport entre démocratie et État-nation, dans le contexte du débat sur la construction européenne et la prégnance des nationalismes sur le vieux continent. Pour lui, le rapport entre l'État-nation et la démocratie est historique et contingent plutôt que nécessaire, et il en appelle au développement d'une politique démocratique qui serait post-nationale — sans être post-étatique.

3. Näsström, 2007 et 20 II. 
En ce qui concerne le problème des frontières, Habermas s'inscrit dans la tradition contractualiste laquelle, il le reconnaît d'emblée, n'offre pas de réponse purement normative à la question des limites d'une association de citoyens libres et égaux:

Il est, en effet, impossible d'expliquer en termes purement normatifs comment doit se composer la totalité fondamentale des personnes qui se rassemblent pour régler leur vie en commun de façon légitime par les moyens du droit positif. D'un point de vue normatif, les frontières sociales d'une association de sujets de droit libres et égaux sont contingentes. Dans la mesure où le caractère volontaire de la décision de s'engager dans une pratique constituante est une fiction du droit rationnel, c'est, dans le monde tel que nous le connaissons, par la contingence historique et par la factualité des événements - et c'est donc en général par l'issue incontrôlable de conflits violents, guerres ou guerres civiles - que sera choisi celui qui va acquérir le pouvoir de définir les frontières d'une communauté politique ${ }^{4}$.

Nous devrions accepter qu'il n'y a pas de solution normative à ce problème et reconnaître le caractère contingent des frontières. Nous ne devrions surtout pas suivre la position nationaliste selon laquelle le principe d'auto-détermination des nations offrirait une solution de rechange à cette résignation.

Si le républicanisme conforte notre conscience de la contingence de ces frontières, le recours à la nation spontanément constituée, pour réduire cette contingence, peut conférer aux frontières l'aura d'une substantialité simulée et la légitimer par des constructions [fictives] de la provenance nationale. Le nationalisme comble la lacune normative par l'appel à un prétendu «droit » à l'auto-détermination nationale 5 .

On voit ici que, pour Habermas, le nationalisme tente de combler la lacune normative en faisant appel à une histoire qui a un sens et une finalité propres. Mais cette histoire est fictive, une fabrication au service d'un projet politique spécifique. Nous devons résister à ce type de projet, notamment parce que l'unité et l'homogénéité de la nation pré-politique sont le plus souvent problématiques, si bien qu'au mieux la satisfaction d'un projet spécifique d'auto-détermination nationale ne fait généralement que déplacer le problème et créer d'autres minorités insatisfaites.

La position de Habermas tient donc en deux points:

I) Les frontières des États démocratiques doivent être acceptées pour ce qu'elles sont: le résultat d'une histoire contingente, ce qui veut dire aussi accepter le vide normatif qui préside à leur fondement.

4. Habermas, I998, p. I06.

5. Habermas, I998, p. I35, l'italique est de moi. 
2) La seule légitimité qu'on puisse accorder aux frontières d'un État démocratique est le résultat d'un processus rétroactif de légitimation ${ }^{6}$. C’est ex post facto que les frontières, établies au départ par la force, peuvent être légitimées, dans la mesure où les pratiques démocratiques constituent au fil du temps une communauté politique dont on peut reconnaître la valeur morale. Inversement, on peut concevoir une contestation des frontières lorsque l'État ne respecte pas les droits et principes démocratiques fondamentaux (p. ex. dans le cas du colonialisme, de violation systématique des droits d'individus ou de groupes, etc.), ou encore lorsqu'aucun effort n'est fait pour tenter de répondre aux revendications légitimes des groupes minoritaires.

Habermas esquisse dans ces textes une position similaire à celle défendue par nombre d'auteurs libéraux qui considèrent que si la constitution des États démocratiques actuels a été le produit de forces moralement arbitraires, ceux-ci ont acquis progressivement une valeur morale qui les rend dignes de respect ${ }^{7}$. Si ces États constituent des espaces de solidarité qui permettent à leurs membres d'accéder à un ensemble important de droits et de bénéfices, ils méritent d'être défendus en l'absence de solution plausible. Des régimes de citoyenneté stables «promote internal redistribution and support co-governance ${ }^{8}$; leur fragilisation ne saurait être un gage de progrès.

\section{Sejeter dans les bras de l'histoire?}

Selon Sofia Näsström, la position conventionnelle revient à mettre les limites

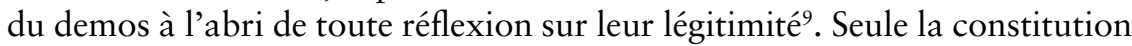
du gouvernement serait ainsi susceptible de faire l'objet d'une interrogation sur sa légitimité alors que la question préalable de la constitution du demos lui-même ne pourrait être soumise à un tel questionnement. Ce faisant, la position conventionnelle nous invite, ni plus ni moins, à nous « jeter dans les bras de l'histoire " plutôt que de laisser ouverte la possibilité de contester la légitimité des frontières déjà données.

Qu'est-ce qui justifie cette caractérisation paradoxale de la position conventionnelle?

«By making the people contingent on history, liberal and deliberative theorists $[\ldots]$ give free reign to the historical forces they seek to exclude $[\ldots]$ this democratic withdrawal indirectly works to the advantage of the status quo. It bene-

6. Nasström, 2007, p. 632.

7. Voir Christiano, 2006; Moore, 2006; Gould, 2006; Kymlicka, 200I. Cara Nine montre, par ailleurs, que ce n'est pas parce que les frontières étatiques sont le produit d'une histoire injuste qu'elles sont pour autant arbitraires d'un point de vue moral (Nine, 2008).

8. Shachar 2009 , p. 67.

9. Näsström 2007 et 20 I I. 
fits those who possess power, status, and force by lending them the opportunity to operate freely in the world without any demands of legitimacy in return. ${ }^{10}$ »

Accepter sans plus les frontières données par une histoire considérée comme contingente a pour effet de consacrer ces frontières, de leur donner un statut non contestable, autrement dit de leur donner une légitimité par défaut. Cela n'est pas acceptable d'abord parce que le statu quo ainsi consacré n'est pas neutre, mais favorise certains groupes particuliers au détriment d'autres groupes. En d'autres termes, l'histoire ne peut pas être utilisée comme un donné, une ligne de fond neutre.

Ensuite, cette position est inacceptable parce que les frontières ne sont pas simplement le résultat d'événements passés, elles ont des effets dans le présent, lesquels doivent pouvoir faire l'objet d'une interrogation sur leur légitimité ou leur justification. Pour Näsström, la constitution du peuple n’est pas simplement ou d'abord un événement historique appartenant au passé, "it is an ongoing claim that we make ${ }^{11}$ ».

Enfin, la position conventionnelle a des conséquences pratiques concrètes, notamment concernant l'immigration. Il en résulte que le débat sur l'immigration se fait dans des termes où la légitimité de la frontière, de la ligne de partage entre membres et non-membres n'est jamais remise en question, et où ce sont nécessairement les premiers qui ont tout pouvoir de statuer sur le sort des seconds.

«The result is that the discussion of political membership - of inclusion and exclusion - takes place against the backdrop of a people that itself remains unquestioned. Migration is the problem, whereas the people is the one that resolves it. ${ }^{12}$ »

Näsström entend contester ce rapport d'asymétrie entre citoyens et migrants. Cela veut dire ne pas accepter la factualité des frontières comme l'arrière-plan nécessaire et légitime des pratiques démocratiques. Loin de soustraire la question du demos à toute exigence de justification, il nous faudrait plutôt rechercher les mécanismes démocratiques appropriés à travers lesquels on puisse "promouvoir» cette contestation.

L'argument de Näsström devrait conduire les partisans de la position conventionnelle à assumer pleinement les conséquences de leur recours à l'histoire: si la constitution du demos repose sur une lacune normative, les frontières spécifiques d'un demos demeurent ouvertes à la contestation puisque leur justification dépend de la façon dont l'État satisfait, effectivement, les exigences de la justice démocratique. La portée de cette justice s'étend non seulement aux citoyens, mais aussi aux non-citoyens dans leurs rapports à l'État démocratique.

10. Näsström 2007, p. 646.

11. Ibid., p. 645 .

12. Ibid., p. 648 . 
Le recours à l'histoire ne doit pas être un moyen d'éviter ou de forclore ces contestations. Il doit être plutôt le point de départ établisitué d'un débat concernant la façon dont un État démocratique spécifique prétend exercer le contrôle de l'accès à son territoire, débat auquel doivent pouvoir participer ceux-là même qui contestent leur exclusion: migrants, demandeurs d'asile, sans-papiers. Autrement dit, si les frontières établies à l'origine par la force ne peuvent être légitimées qu'ex post facto, ce processus de légitimation rétroactif doit inclure non seulement les citoyens, mais aussi les non-citoyens concernés. Il n'est sans doute pas possible ni souhaitable d'atteindre un rapport de parfaite symétrie entre citoyens et non-citoyens, mais le refus de reconnaître aux étrangers toute voix au chapitre n'est pas justifiable.

\section{Dissoudre le problème de la frontière? La position cosmopolitique}

Certains auteurs contestent toutefois que la recherche de la raison et du droit doive s'arrêter aux frontières territoriales des États et récusent l'idée même que la théorie démocratique soit incomplète et n'offre pas de réponse normative au problème des frontières. Si l'on poussait à leur terme les principes fondateurs de la théorie démocratique, on s'apercevrait que la réponse proprement normative à laquelle ils nous mènent est celle du demos global. Il n'y a en fait de "lacune normative" que parce que nous tenons à l'idée que le demos a nécessairement des limites spécifiques, bien que nous soyons obligés de constater que les principes démocratiques ne permettent pas de les justifier. Nous en inférons alors que la théorie de la démocratie est incomplète et nous nous tournons vers l'histoire. Mais si nous abandonnions ce présupposé et si nous allions au bout de ce qu'impliquent les principes démocratiques de légitimité, nous serions à même de constater que le problème des frontières est un faux problème. Nous le verrions se dissoudre devant nos yeux: à la question sans réponse des limites du demos, nous pourrions opposer le principe d'un demos sans limite. Il existe deux versions de cette position, lesquelles se distinguent, pour l'essentiel, par la façon dont le principe démocratique de légitimité est défini: la première s'appuie sur le principe du "all affected interests ${ }^{13}$ ", tandis que la seconde mise plutôt sur le principe $\mathrm{du}$ "all those subjected ${ }^{14}{ }^{»}$.

\section{Le principe du «all affected interests »}

Robert Goodin cherche une issue au problème de circularité identifié par Whelan en partant de cette hypothèse: s'il nous est possible de contester le caractère démocratique de la constitution d'un demos donné, c'est qu'il doit exister au moins un principe démocratique auquel nous faisons implicitement référence lorsque nous formulons cette critique ${ }^{15}$. Pour le mettre en

13. Arrhenius, 2007; Goodin, 2007.

14. Abizadeh, 2008.

15. Goodin, 2007, p. 47. 
évidence, il nous propose d'examiner les bases à partir desquelles on considère que les demoï se sont généralement constitués, soit la territorialité, la nationalité ou l'histoire. Pour Goodin, ces facteurs sont tous également arbitraires d'un point de vue moral. Ils ne peuvent être invoqués comme des raisons permettant de justifier une délimitation spécifique du demos que dans la mesure où ils conduisent à l'enchevêtrement («intertwining») des intérêts des individus ${ }^{16}$. Ainsi, nous pensons que les groupes territoriaux, nationaux, historiques devraient exercer un pouvoir collectif de décision parce que, de façon générale, les intérêts des individus au sein de ces groupes sont affectés par les actions et les choix des autres individus qui y appartiennent, et réciproquement.

La corrélation territorialité-nationalité-histoire, d'une part, et intérêts partagés, d'autre part, n'est pas parfaite. Goodin en infère que constituer un demos sur la base d'un territoire partagé, d'une histoire ou d'une nationalité communes n'est jamais qu'une approximation imparfaite de ce que donnerait le fait de le former sur la base de la présence d'intérêts entremêlés. Ainsi, on peut facilement évoquer plusieurs cas empiriques où l'approximation est soit trop inclusive (y compris dans le demos des individus dont les intérêts ne sont pas affectés par ses décisions, que ce soit pour une décision en particulier ou de manière générale), soit trop exclusive (parce qu'elle n'inclut pas des individus dont les intérêts, au contraire, sont touchés). Mais se référer à de tels contre-exemples, c'est déjà supposer que «the all-affected interests is the principle that is being implicitly employed in judging all those other methods of constituting the demos, on the basis of territoriality, nationality, history, or whatever». Par conséquent, conclut-il: "The "all affected interests" principle is the standard by which adequacy of those other approximations is invariably assessed ${ }^{17}$.»

C'est donc à ce principe du "all affected interests" que conduirait l'analyse de nos jugements concernant le caractère plus ou moins approprié des demoï existants. Goodin entend dégager ce principe de ses scories empiriques pour déterminer quelles seraient ses implications si nous l'appliquions aujourd'hui de façon cohérente. Mais il faut au préalable en proposer une interprétation déterminée, en l'espèce la version "possibiliste ${ }^{18}$ » qui donne au principe son extension maximale en stipulant que toutes les personnes "possiblement» affectées par une décision donnée doivent pouvoir participer à la prise de décision collective. Puisque les décisions collectives peuvent avoir des conséquences indésirables et que leur effet est potentielle-

16. Ibid., p. 48 .

17. Goodin, 2007 , p. 49.

18. Celle-ci se distingue des versions «all actually affected " et "all plausibly affected" critiquées par Goodin. Held propose plutôt "all significantly affected», soit "people [...] enmeshed in decisions and forces that impact on their capacity to fulfill their vital needs » (Held 2005 , p. I4). 
ment infini, il est impossible de spécifier tous ceux qui pourraient être affectés par une décision déterminée, sauf si l'on inclut au sein du demos la planète toute entière, y compris les générations futures. L'application cohérente du principe conduirait donc à considérer que le demos global est la réponse donnée par la théorie normative de la démocratie à la question des limites du demos ${ }^{19}$. Goodin reconnaît que sa proposition semblera - au mieux - difficilement réalisable. Cela étant, il accepte que nous retrouvions le chemin des faits, non pas toutefois pour nous satisfaire de la situation présente, mais plutôt pour réfléchir aux arrangements qui nous permettraient le mieux de nous rapprocher de l'idéal, malgré la persistance d'États démocratiques territoriaux.

La façon dont Goodin dérive son principe normatif est-elle convaincante? Trouve-t-il ainsi une issue au problème de la circularité mis en évidence par Whelan et Dahl ? Remarquons d'abord le glissement qu'il effectue entre le constat de l'importance du facteur d'enchevêtrement des intérêts, ce qu'il appelle le "principe» des «entertwined interests », et celui des «affected interests ». Il semble supposer que les deux sont équivalents, ce qui n'est pas le cas. En effet, l'enchevêtrement d'intérêts renvoie plutôt à l'existence d'un monde commun où tous, ou presque tous les interêts fondamentaux des individus sont mutuellement impliqués ${ }^{20}$. L'extension d'un tel "principe" est beaucoup plus limitée que celle appelée par le principe de «tous les intérêts affectés ». Suivant ce dernier, en effet, il suffit que l'action d'un État affecte (possiblement) l'un de mes intérêts pour que je puisse considérer avoir le droit de prendre part à cette décision. On peut considérer, à l'instar de Goodin, que l'application d'un tel principe dans notre monde globalisé signifierait (nous pouvons tous être possiblement affectés par la décision d'un État donné), que nous devons adhérer à l'idée que toute limitation du demos est illégitime. Mais ce n'est pas le cas du «principe» des intérêts enchevêtrés.

En outre, je mets «principe» entre guillemets parce que l'enchevêtrement des intérêts est moins un principe normatif nous donnant les moyens de distinguer entre les demoï légitimement constitués et ceux qui ne le sont pas qu'une explication nous permettant de comprendre pourquoi, au fil du temps, des collectivités qui ont été constituées souvent dans le sang et la guerre finissent par acquérir une valeur morale aux yeux de leurs membres alors même que certains d'entre eux sont les lointains descendants des vaincus du passé.

Ainsi, on ne saurait prétendre que l'enchevêtrement des intérêts constitue un principe normatif de constitution du demos, ni l'identifier au principe de «tous les intérêts affectés ". Cela étant, Goodin ne peut ni affirmer que le principe du «all affected interests» serait contenu in nuce

19. Goodin, 2007 , p. 55 et p. 64 .

20. Christiano, 2006, p. 98. 
dans nos jugements sur le caractère approprié ou non des frontières de demoï existants, ni prétendre qu'appliqué de façon cohérente ce principe nous contraindrait à reconnaître que le demos global est la seule réponse légitime à la question de la constitution du demos. L'interprétation extensive qu'il fait de ce principe conforte ce que Whelan et Dahl avaient déjà montré, à savoir que sa portée est indéfinie. Mais on ne saurait transformer l'indétermination d'un principe en réponse déterminée (le demos global) au problème de la constitution du demos ${ }^{21}$.

\section{Pouvoir de contrainte et droit de participation}

Dans une contribution importante dont l'objet premier est de montrer que les États démocratiques n'ont pas droit au contrôle unilatéral de leurs frontières, Arash Abizadeh prend pour point de départ le principe démocratique selon lequel l'exercice du pouvoir de contrainte n'est légitime que s'il est justifié auprès de tous ceux sur lesquels il s'exerce ${ }^{22}$. Il ajoute que, du point de vue d'une théorie démocratique, l'exigence de justification suscitée par l'exercice de la contrainte ne peut pas être comprise comme simplement hypothétique - ce que suggère la tradition libérale —, mais qu'elle implique plutôt un droit effectif de participation ${ }^{23}$. Dans la mesure où un régime de contrôle des frontières soumet tant les membres que les non-membres à l'exercice du pouvoir coercitif de l'État, un tel régime doit être justifié tant pour ses citoyens que pour les étrangers, ce qui implique de donner à ces derniers les moyens institutionnels de faire entendre leur voix.

Selon Abizadeh, on ne saurait tenir pour acquis que le «tous ceux» de la prémisse ( «l'exercice du pouvoir de contrainte n'est légitime que s'il est justifié auprès de tous ceux sur lesquels il s'exerce ") réfère aux seuls citoyens plutôt qu'à l'ensemble de ceux qui sont effectivement soumis à la contrainte, qu'ils soient ou non citoyens. Il n'y a pas de raison soutenable de limiter ainsi d'emblée la portée de l'exigence de justification. Au contraire, la théorie de la démocratie donne une réponse au problème de la frontière de Whelan: l'extension du principe de légitimité démocratique s'étend aussi loin que nos pratiques de justification mutuelle peuvent aller, ce qui veut dire que le demos est en principe sans limite ${ }^{24}$.

Comment comprendre cette thèse? À première vue, elle apparaît comme énonçant un principe régulateur, formulant une exigence morale, qui nous motive à éprouver jusqu'à quel point les institutions d'un demos réellement

21. Pour des critiques qui touchent les problèmes d'opérationnalisation du principe, voir Karlsson Schaffer, 20I 2, et Nootens, 2009.

22. Abizadeh, 2008, p. 4I.

23. Nous devrions comprendre ce droit: «in whatever sense is required for persons to see themselves as the free and equal authors of the laws to which they are subject " (Abizadeh 2008, p. 4I).

24. Abizadeh 2008, p. 48. 
existant satisfont ou non aux exigences de la légitimité démocratique, et à accepter que nous devons tenter d'étendre nos pratiques de justification mutuelle au delà du seul corps des citoyens pour atteindre l'ensemble des individus soumis au pouvoir coercitif que représentent les frontières de l'État démocratique. Considérée de ce point de vue, la thèse d'Abizadeh semblera très proche de la position de Näsström, mais reformulée dans un vocabulaire différent, à saveur cosmopolitique.

Mais Abizadeh donne également une version plus forte des implications de sa position:

Nous devrions, comme démocrates, nous reconnaître contraints, par notre propre conception de la légitimité politique, à soutenir la création d'institutions démocratiques cosmopolitiques ayant juridiction sur les conditions d'entrée sur le territoire étatique. Si toute frontière d'un État, en tant qu'elle limite la liberté de mouvement de tout individu, le soumet à un pouvoir coercitif qui suscite une exigence de justification, si la satisfaction de cette exigence requiert la reconnaissance d'un droit de participation au processus par lequel le régime de la frontière est déterminé, cela signifie non seulement que toute frontière existante est illégitime, mais surtout que seules des institutions démocratiques globales pourraient constituer un cadre adéquat pour déterminer un régime légitime de contrôle des frontières. Dans la mesure où, pour tout État exerçant le contrôle de ses frontières, on peut montrer que l'ensemble de ceux à qui une justification est due recouvre chaque fois l'ensemble de l'humanité, la création d'institutions démocratiques globales s'avère nécessaire.

Cette position repose sur deux points controversés: premièrement, une conception extensive de ce qui constitue l'exercice d'un pouvoir de contrainte; deuxièmement, l'idée que l'exigence de justification doive prendre la forme d'un droit effectif de participation.

Selon Abizadeh, un individu est soumis à un pouvoir de contrainte dès lors que les agents de l'État sont capables d'utiliser la force physique (ce qui inclut tant le recours à la force que le fait d'ériger des obstacles physiques à la liberté de mouvement) et autorisés à le faire, ou de menacer d'en faire usage de façon à empêcher ou prévenir certains comportements. Les individus sont soumis à cette contrainte, que les agents de l'État réussissent ou non à prévenir ou empêcher ces comportements, et indépendamment des intentions des individus. Même si je n'ai jamais eu l'intention de franchir la frontière de l'État " $\mathrm{x}$ ", le fait que son accès soit limité par l'exercice du pouvoir de contrainte de cet État suffit, selon Abizadeh, pour qu'il soit possible de dire que je suis soumise à ce pouvoir. Le contenu de mon intention ne change rien au caractère contraignant du pouvoir ${ }^{25}$.

Il est juste de dire que la loi qui prévoit l'interdiction de fumer dans les lieux publics conserve un caractère contraignant, même si je suis non fumeuse et n'ai nulle intention de fumer. Par contre, pour pouvoir prétendre

25. Abizadeh, 2008, p. 57-60. 
que cette loi a un caractère contraignant pour moi, il faut pouvoir mettre en évidence un lien quelconque - qui n'a pas à être de l'ordre de l'intention - entre l'individu que je suis et cette loi (ce peut être, par exemple, que je suis citoyenne ou de passage dans cet État). En l'absence d'un tel lien, formel ou informel, je peux reconnaître que cette loi a un caractère contraignant au sens où c'est là un attribut généralement associé à toute loi, sans prétendre pour autant qu'elle me soumet à la contrainte. Si c'était le cas, cela reviendrait à dire que les lois du Vanuatu me soumettent à un pouvoir coercitif du simple fait que ce sont des lois; c'est peut-être juste si l'on accepte la définition qu'Abizadeh donne de la contrainte, mais c'est là une proposition trop faible pour pouvoir donner naissance à une exigence de justification qui implique un droit de participation ${ }^{26}$.

Cette définition de la contrainte fait en sorte que tout régime de frontière - pour autant qu'il présente un obstacle à la liberté de mouvement de tout agent - constitue une atteinte à son autonomie, laquelle donne naissance à une exigence de justification, peu importe ce que signifie pour cet agent cette restriction à sa liberté. En d'autres termes, on n'a pas à considérer l'effet spécifique qu'a effectivement cette restriction pour cet agent. Cela veut dire que l'on peut parler de coercition et d'atteinte à l'autonomie aussi bien dans le cas d'un citoyen canadien, invité dans une université brésilienne, et qui se voit refoulé à son arrivée au Brésil parce qu'il n'a pas de visa, que dans le cas de travailleurs mexicains sans papiers qui vivent et travaillent dans un État américain dans la peur de l'expulsion. Et pourtant, l'effet de l'obstacle que représente la frontière sur leurs vies respectives est très différent. Ces différences substantielles entre les deux situations ne devraient-elles pas avoir un effet sur les exigences qu'elles peuvent susciter?

Rappelons de nouveau que, pour Abizadeh, reconnaître la légitimité d'une exigence de justification, c'est reconnaître un droit de participation effectif dans des institutions communes. C'est peut-être là surtout que le bât blesse. En effet, on pourrait concevoir que les régimes des frontières constituent une limitation de l'autonomie des individus qui y sont soumis laquelle suscite une exigence de justification - , sans pour autant accepter que cette exigence doive se traduire par un droit effectif de participation pour l'ensemble de ces individus ${ }^{27}$.

La tradition démocratique, à laquelle Abizadeh se réfère, enseigne depuis Rousseau que la loi à laquelle je suis contrainte d'obéir n'est légitime que si je puis me représenter comme son co-auteur. Mais cette prescription

26. Dans sa critique, Miller (20I0) insiste plutôt sur la distinction entre coercition et prévention (voir la réplique d'Abizadeh, 20I0) pour tenter de montrer que les régimes de frontières, contrôlés unilatéralement par les États souverains, ne constituent pas l'exercice d'un pouvoir de coercition sur les non-citoyens. Ce n'est pas ma position: ces régimes constituent, dans certains cas, l'exercice d'un tel pouvoir.

27. Lopez-Guerra, 2005, p. 219; Bauböck, 2009, p. 19 n. 
ne prend son sens que dans le contexte plus large du pacte social lui-même, où les individus engagent chacun leur personne et leurs biens pour former l'association. Autrement dit, le droit de participation n'est pas lié à l'exercice de telle contrainte déterminée, mais à un ensemble de contraintes qui touchent l'ensemble de ma personne et mes biens. Si l'on peut dégager du principe de légitimité démocratique un droit effectif de participation, celuici ne concerne que les individus soumis à l'ensemble, ou, du moins, à une part significative, de la gamme de lois contraignantes de l'association, non pas ceux qui ne subissent que l'une ou l'autre d'entre elles. Si mon analyse est juste, cela voudrait dire, par exemple, que si les sans-papiers, les travailleurs saisonniers, les immigrants qui sont soumis à un ensemble significatif de contraintes de l'État démocratique peuvent revendiquer de façon plausible un droit de participation aux processus démocratiques au travers desquels ce dispositif de contraintes est déterminé, cela n'est pas le cas pour l'ensemble des non-citoyens. Ainsi, le principe de légitimité démocratique ne mène pas au demos global comme solution au problème des frontières. L'idée de demos sans limite, comme principe régulateur, a une portée indéfinie et ne donne, par conséquent, aucune réponse déterminée à la question de la constitution du demos. Il nous engage cependant à vérifier jusqu'où effectivement nous devons étendre nos pratiques de justification mutuelle afin de répondre aux revendications de ceux qui pâtissent, concrètement, de l'existence de frontières dont nous reconnaissons les origines contingentes.

\section{Condusion}

En acceptant que les limites du demos soient données généralement par une histoire dont nous reconnaissons la contingence, ne renonçons-nous pas trop tôt à la recherche du droit et de la raison pour nous résigner aux faits ? Nous pouvons maintenant répondre non à cette question, mais seulement pour autant que nous acceptions de tirer pleinement les conséquences de ce recours à l'histoire. Celui-ci ne doit pas se muer dans la consécration de frontières incontestables, pas plus qu'il ne doit sanctionner le droit des citoyens des États démocratiques du Nord à rester sourds aux revendications de ceux qui frappent à leurs portes afin d'améliorer leur sort. Si la question de la légitimité du demos ne peut se poser qu'a posteriori et porter sur les conditions auxquelles ses limites pourraient être justifiées ${ }^{28}$, alors nous devons reconnaître que cette interrogation doit tenir compte de la façon dont le demos traite, non pas seulement ses propres citoyens, mais aussi ceux qui, sans être citoyens, partagent avec eux un ensemble complexe de relations de dépendance mutuelle. 


\section{Références}

Abizadeh, Arash. «Democratic Theory and Border Coercion. No Right to Unilaterally Control Your Own Borders ", Political Theory, 36 (I), 2008, 37-65.

_. "Democratic Legitimacy and State Coercion: A Reply to David Miller ", Political Theory 38 (I), 2010, I2I-I30.

Arrhenius, Gustaf. "The Boundary Problem in Democratic Theory », Draft 0703 I 2, http://people.su.se/ folke/boundary.pdf

Bauböck, Rainer. "The Rights of Others and the Boundaries of Democracy ", European Journal of Political Theory, 6 (4), 2007, 398-405.

—. "Global Justice, Freedom of Movement and Democratic Citizenship ", European Journal of Sociology, 50 (I), 2009, I-3I.

Blake, Michael. "Distributive Justice, State Coercion, and Autonomy », Philosophy and Public Affairs, 30 (3), 2002, 257-295.

Christiano, Thomas. "A Democratic Theory of Territory and Some Puzzles about Global Democracy ", Journal of Social Philosophy, 37 (I), 2006, 8I-I07.

Dahl, Robert A. I970. After the Revolution? Authority in a Good Society, (revised ed. I990) New Haven \& London, Yale University Press.

- Democracy and its Critics, New Haven \& London, Yale University Press, I989.

Goodin, Robert E. «Enfranchising All Affected Interests, and its Alternatives », Philosophy and Public Affairs, 35 (I), 2007, 4I-67.

Gould, Carole. Globalizing Democracy and Human Rights, Cambridge, Cambridge University Press, 2004.

Habermas, Jürgen. L'intégration républicaine, Paris, Fayard, I 998.

Held, David. "Principles of Cosmopolitan Order », The Political Philosophy of Cosmopolitanism, G. Brock, H. Brighouse (dir.), Cambridge, Cambridge University Press, 2005, I0-28.

Karlsson Schaffer, Johan. "The Boundaries of Transnational Democracy: Alternatives to the All-Affected Principle ", Review of International Studies, 38 (2), 2OI 2, 32I-342.

Kymlicka, Will. Politics in the Vernacular. Nationalism, Multiculturalism, and Citizenship, Oxford, Oxford University Press, $200 \mathrm{I}$.

Miller, David. "Democracy's Domain », Philosophy and Public Affairs 37(3), 2009, 2OI-228.

—. "Why Immigration Controls are not Coercive: A Reply to Arash Abizadeh» Political Theory 38 (I), 2010, I I I-I 20.

Moore, Margaret. "Cosmopolitanism and Political Communities ", Social Theory and Practice, 32 (4), 2006, 627-658.

Näsström, Sofia. "The Legitimacy of the People», Political Theory 35 (5), 2007, 624-658.

- "The Challenge of the All-Affected Principle», Political Studies 59, 20I I, I I6-I34.

Nine, Cara. "The Moral Arbitrariness of State Borders: Against Beitz ", Contemporary Political Theory, 7 (3), 2008, 259-279.

Nootens, Geneviève. "Democracy and Legitimacy in Plurinational Societies", Contemporary Political Theory, 8 (3), 2009, 276-294.

Rousseau, Jean-Jacques. (Euvres complètes III. Du contrat social. Écrits politiques, Paris, Gallimard (La Pléiade), I964. 
Shachar, Ayelet. The Birthright Lottery. Citizenship and Global Inequality, Cambridge \& London, Havard University Press, 2009.

Whelan, Frederick. "Prologue: Democratic Theory and the Boundary Problem", Nomos XXV: Liberal Democracy, ed. J. Rolan Pennock, John W. Chapman, New York \& London, New York University Press, I983. 